\title{
Silencing of CD44 in glioma leads to changes in cytoskeletal protein expression and cellular biomechanical deformation properties as measured by AFM nanoindentation
}

Zaynah Maherally*, James R. Smith, Manar K. Ghoneim, Luke Dickson, Qian An, Helen L. Fillmore and Geoffrey J. Pilkington*

Cellular and Molecular Neuro-oncology Research Group, Institute of Biomedical and Biomolecular Sciences, School of Pharmacy and Biomedical Sciences, University of Portsmouth, St Michael's Building, White Swan Road, Portsmouth PO1 2DT, UK

Running Title: CD44 silencing leads to cytoskeleton changes

*For correspondence. Tel: +44 (0)23 9284 2046, Fax: +44 (0)23 9284 3565;

E-mail: Zaynah.maherally@port.ac.uk and Geoff.pilkington@port.ac.uk

\begin{abstract}
CD44, a transmembrane glycoprotein receptor for extracellular matrix molecules such as hyaluronic acid and osteopontin, is involved in glioma cellular signalling, adhesion and invasion. Although a great deal is known concerning the molecular players in adhesion, migration and invasion, little is known relating to how these invasive and migratorypromoting proteins influence biomechanical properties of glioma cells. Herein, we extend previous CD44 blocking experiments to examine effects of CD44 knock-down on expression of cytoskeletal proteins and cellular stiffness.

An atomic force microscope (AFM) nanoindentation method was used to measure deformation or cellular stiffness (Young's Modulus, $E$ ) in real time, at the single cell level over nuclear and cytoplasmic regions. A glioblastoma cell line (SNB-19) was transfected with either CD44 siRNA, scrambled siRNA, or a non-related gene siRNA. In SNB-19 CD44 knock-down cells, levels of microtubule, vimentin and GFAP proteins were lower compared to cells transfected with scrambled siRNA. Functionally, CD44 knock-down cells were less migratory compared to controls. AFM nanoindentation results show that the area over the nuclei of both knock-down and parental control cells examined were significantly more compliant than their cytoplasmic regions $(p<0.001)$. The most striking difference was seen when comparing nuclear regions of parental control cells versus CD44 knock-down cells. CD44 knock-down SNB-19 cells $(E=0.56 \pm 0.50 \mathrm{kPa})$ were less stiff than parental cells $(E$ $=1.93 \pm 2.86 \mathrm{kPa} ; p<0.001)$. Based on these results we hypothesise that CD44 signalling via cytoskeletal proteins such as vimentin may influence the ability of glioma cells to respond to host-tumour derived mechanical pressures.
\end{abstract}

Keywords: Glioma; migration; cytoskeleton; cell stiffness; atomic force microscopy (AFM) 
BioNanoScience

First online: 26 December 2015, pp. 1-11, doi: 10.1007/s12668-015-0189-2

www.link.springer.com/article/10.1007/s/12668-015-0189-2

\section{Introduction}

Glioma cell invasion is strongly influenced by multiple modes of interactions including tumour cell/host cell communication and signalling and biophysical properties of both tumour cell and extracellular matrix (ECM) [1,2]. The glioma microenvironment is dynamic in nature and, in response to therapies, will adapt to survive. While our understanding of host/tumour, immune/tumour interactions are improving, the appreciation of how biomechanical and biophysical properties contribute to influencing glioma growth and invasion is beginning to be recognised $[1,3]$. Identification of key host/tumour interactions and the integration of how mechanical cues drive tumour adaptations could provide potential targets involved in therapeutic resistance. Mechanical cues that can potentially influence the invasive process include substrate stiffness, fluid shear flow and local and global cell strain due to host/tumour changes (i.e., growth, edema). How a glioma cell responds to mechanical cues will most likely depend on its genomic, epigenomic, and proteomic composition as well as ECM stiffness. It will be important to understand the integration of biophysical information during changes associated with glioma growth and during therapeutic challenges in terms of understanding adaptive mechanisms [2]. The ability of a cell to deform is related to cytoskeletal components, actin, microtubulin, and intermediate filaments $[4,5]$. The degree to which particular cytoskeletal component is more important may be dependent on experimental systems [5]. There is a documented association of mechanical phenotype with cancer development; however specific mechanisms are yet to be agreed upon $[4,6,7]$. Recent studies on the dynamic reorganisation of the cytoskeleton in cancer cells have drawn great interest in understanding mechanical properties involved in cancer invasion and metastasis [4,8-10]. Changes in such biomechanical properties and cytoskeletal reorganisation have been observed in cancer cells of aggressive phenotypes [4,10]. Indeed, it has been suggested that the elastic properties (i.e., stiffness) of tissue/cells could be an indication of cancer $[11,12]$, and previous studies using different biomechanical assays have revealed a correlation between reduced stiffness and increased metastatic potential of human cancer cells [13].

CD44 is a transmembrane receptor expressed on glioma cells and is known to be involved in cell adhesion, glioma invasion and migration [14-18]. CD44 serves as a cell receptor for hyaluronic acid $[20,21]$ and osteopontin [20]. Hyaluronic acid (HA), a glycosaminoglycan [21], is a major component of brain ECM and high levels, corresponding to increased malignancy, are expressed in tumours [22-24]. HA binds to the surface of GBM cells via CD44, a cell adhesion glycoprotein [26], which is essential for the maintenance of the threedimensional structure of organs/tissues; CD44 is present in many cells including normal astrocytes, but is significantly upregulated in GBM [26]. Recently, it has been reported that CD44 expression correlates with short survival in the proneural subtype of GBM [20]. We have previously described that inhibition of CD44 expression in glioma cell lines, either by antibody blocking or gene silencing (siRNA), produced GBM cells that lacked invadopodia, were rounder and less invasive than wild type cells [27]. This effect was mediated in part by RhoGTPase signalling [27]. This signalling pathway has been implicated in cellular stiffness in U373 glioma cells [28]. In the current paper, we report the effects of CD44 knockdown in glioma cell lines with an emphasis on the examination of cytoskeletal protein expression and cellular stiffness measured using atomic force microscopy (AFM). This technology allows determination of the ability of a cell to deform through the analysis of force vs. displacement curves, obtained by placing the AFM probe over the cell and making nanoindentations. This measure of deformity or elasticity is known as the elastic modulus or Young's modulus $(E)[29,30]$. While discovered as a means to examine different types of materials, $E$ is classically defined as the ratio of stress (force / area) along an axis to strain 
(deformation) in which Hooke's law (restoring force to a spring is proportional to a small displacement) holds [31,32]. AFM nanoindentation studies have been performed on a variety of cell types to obtain $E$ values, which typically range from $100 \mathrm{~Pa}$ (epithelial, compliant) [33] to $100 \mathrm{GPa}$ (enamel, stiff) [34]. Such measurements have also been carried out on a broad range of cancer cells and tissues, including bladder $[35,36]$, breast $[6,37-$ 40], cervical [41], esophageal [42], liver [43,44], lung [37], ovarian [4,45], pancreas $[37,46,47]$, prostate $[6,48,49]$, thyroid [50] and melanoma [10]. In most studies, AFM measurements were usually performed by probing specific regions of the cell, such as central $[37,38,48]$, above the nucleus $[4,6,10,41,42,49]$, or perinuclear regions $[45,49]$. Typically, cancer cells are less stiff (lower $E$ ) compared to their benign counterparts. It has been suggested that softer cells are more motile than stiffer cells and this has been linked to the ability of malignant cells to invade and metastasise in vivo $[4,7,35,37,45]$. In this paper, we report on the effects of CD44 knock-down in glioma cell lines with an emphasis on the examination of specific cytoskeletal protein expression and cellular stiffness $(E)$ obtained from AFM measurements.

\section{Materials and Methods}

\subsection{Cells}

The established human glioblastoma cell line, SNB-19 (passage-44), was obtained from the DSMZ German Brain Tumour Bank (Germany). The cell lines were authenticated using an STR-PCR kit, GenePrint 10 (Promega, UK), as per the manufacturer's instruction. The STR profile of the cells was compared with the DSMZ database to confirm the identity of the SNB-19 cells. Cells are routinely mycoplasma tested utilizing a kit from Lonza, Germany. Cell authentication is also routinely checked using a microfluidic electrophoresis system incorporating an Agilent 2100 Bioanalyzer (Agilent Technologies, USA) to analyse STR-PCR fragments from 10 human genomic loci of human cell lines [51]. CD44 knock-down in SNB19 cells was achieved by CD44-targeting SMARTpool ${ }^{\circledR}$ siRNA transfection (Dharmacon, UK), following the manufacturer's instructions [27]. Briefly, SNB-19 cells were incubated with CD44-siRNA for $96 \mathrm{~h}$ to achieve $>80 \%$ knock-down of the target gene and for $120 \mathrm{~h}$ in morphology assays. An Accell ${ }^{\mathrm{TM}}$ non-targeting pool siRNA was included in the knock-down experiments as the negative control and GADP-siRNA as the positive control. The GADPsiRNA also served to optimise and monitor efficiency of siRNA delivery into SNB-19 cells [27]. All cells were cultured in Dulbecco's Modified Eagle Medium (Gibco, UK) with 10\% foetal calf serum (Sigma, Dorset, UK).

\subsection{Antibodies}

Primary antibodies. Mouse monoclonal anti-CD44 (1:500 in immunocytochemistry; $1: 25$ in flow cytometry) was purchased from Chemicon (UK). Mouse monoclonal anti-F-actin (1:200 and 1:20) from Invitrogen (UK), mouse monoclonal anti-microtubule (1:100 and 1:10) from Santa Cruz (USA), rabbit polyclonal anti-GFAP (1:200 and 1:20) and mouse monoclonal anti-Vimentin (1:500 and 1:50) both from Dako (UK) were used for immunocytochemistry and flow cytometry, respectively. Rabbit polyclonal anti- $\beta$-actin (1:1000) (Sigma), rabbit polyclonal anti-CD44 (1:250) (Santa Cruz), mouse monoclonal anti-microtubule (1:500) (Santa Cruz), rabbit polyclonal anti-GFAP (1:1000) (Dako), mouse monoclonal antiVimentin (1:1000) (Dako) and goat polyclonal anti-GAPDH (1:200) (Abcam) were used for Western blotting. 
Secondary Antibodies. Fluorochrome-conjugated AlexaFluor-488 (Invitrogen) was used in flow cytometry and ICC both at dilution 1:500. Horseradish peroxidase (HRP)-conjugated IgG (Invitrogen) was used for chemiluminescent detection in Western blotting $(1: 1000)$.

\subsection{Immunocytochemistry (ICC)}

ICC was carried out following an established procedure previously described [27]. Cells were fixed with $4 \%$ paraformaldehyde (PFA) (Sigma, UK) and permeabilised with $0.2 \%$ Triton X100 (Sigma, UK) for intracellular antigen detection before incubation with the primary antibody. Cells were then incubated with the relevant secondary antibody. Cells were washed with $1 \times$ PBS before and after each antibody's incubation. Nuclei were counterstained with Hoechst Blue (Sigma, UK) and the slides were viewed using a Zeiss Axio Imager Z1 fluorescence microscope (Carl Zeiss, Welwyn Garden City, UK). Images were captured using Volocity Image Analysis Software (V5.2, Perkin Elmer, Cambridge, UK).

\subsection{Flow cytometry}

Flow cytometry was performed based on standard protocols [27]. Briefly, cells were permeabilised with cytofix/cytoperm solution (BD Biosciences, UK) for intracellular antigen staining prior to primary antibody incubation. Cells were then washed with $5 \%$ serum/PBS and incubated with the corresponding secondary antibody. After the incubation, cells were washed and resuspended in 1\% serum/PBS then transferred to fluorescence-activated cell sorting (FACS) tubes (BD Biosciences) for analysis. Each sample was analysed in triplicate plus one negative control where primary antibody was omitted, and the experiment was repeated three times. Analysis was performed on a four-colour-multi-parameter FACS Calibur (BD Biosciences) and the expression level was assessed by the percentage of positive cell population [positive cells (\%)], as described previously [28].

\subsection{Western blotting (WB)}

Cell lysates were separated in the "any kDa" pre-cast SDS-PAGE gel (Bio-rad Laboratories Ltd, UK) and transblotted onto a polyvinylidene difluoride membrane (GE Healthcare Life Sciences, UK). Immunodetection was achieved using the primary antibodies and horseradish peroxidase-conjugated secondary antibodies. The blot was then visualised and analysed with the GBOX Chemi XT16 system (Synoptics). Parental SNB-19 cells and nontargeting siRNA treated cells were used as controls in various assays (refer to individual results and figure legends). For siRNA knock-down experiments, all transfected cells were harvested after $96 \mathrm{~h}$ siRNA-incubation for lysate extraction.

\subsection{Migration assay}

Cell migration was assessed through the Transwell ${ }^{\mathrm{TM}}$ modified Boyden Chamber assay. Cells were grown in serum free media. The Transwell ${ }^{\mathrm{TM}}$ inserts used were $8 \mu \mathrm{m}$ porosity, polycarbonate membrane filters within 24-well plates (Corning, UK). Platelet-derived growth factor $\left(P\right.$ GGF $_{A B} ; 10 \mathrm{ng} / \mathrm{ml}$; Universal Biologicals Cambridge, UK) diluted in serum free media (SFM) was used as a chemoattractant and added to the lower chamber of the well. Cells were harvested, counted and seeded at a concentration of $1 \times 10^{5}$ cells $/ 100 \mu \mathrm{l}$ SFM in the upper compartment of the Transwell ${ }^{\mathrm{TM}}$ unit. Migration was allowed to occur for $6 \mathrm{~h}$ at $37^{\circ} \mathrm{C}$ in a $5 \% \mathrm{CO}_{2}$ chamber. The filters were then removed and fixed in methanol. Non-migrated cells on the upper surface of the filter were removed with a cotton swab and migrated cells, adherent on the lower filter surface were stained with Diff Quick (BDH Laboratory Supplies, UK) and cells were counted in 5 random fields. Each experiment was performed in triplicate. 
First online: 26 December 2015, pp. 1-11, doi: 10.1007/s12668-015-0189-2

www.link.springer.com/article/10.1007/s/12668-015-0189-2

Mean values of migrated cells for each point were calculated. Migration was expressed as mean \pm SEM of the number of total cells counted per well.

\subsection{Confocal microscopy}

Confocal microscopy was carried out with a Zeiss LSM 510 Meta Axioskop2 confocal microscope (Carl Zeiss), as previously described [27]. Fluorescence was detected using excitation wavelengths of $488 \mathrm{~nm}$ (green), $568 \mathrm{~nm}$ (red) and $405 \mathrm{~nm}$ (blue), with an argon, HeNe1 and diode laser, respectively. Multi-track image capture was performed with two channels so that different colours were imaged by separate channels to prevent any overlap in excitation spectra. Identical settings were used to capture images of negative controls where primary antibody was omitted.

\subsection{AFM elasticity measurements}

AFM nanoindentation experiments were carried out using a CellHesion 200 AFM (JPK Instruments, Cambridge, UK) mounted on a Zeiss Axiovert 200M (Carl Zeiss) inverted optical microscope placed on a Halcyonics Micro 40 anti-vibration table (Accurion, Goettingen, Germany). A Petri dish of cells (in growth media, lid removed; glass-bottomed) was placed on an integrated Petri dish heater (37 ${ }^{\circ} \mathrm{C}$; JPK Instruments) and a $20 \times$ objective, on the inverted microscope, was used to locate cells of interest. In this experimental design, only single, isolated cells were investigated to avoid influence from neighbouring cells [48]. An 'arrow-shaped', tipless cantilever (Arrow-TL1-50; nominal length $I_{\text {nom }}=500 \mu \mathrm{m}, k_{\text {nom }}=$ $0.03 \mathrm{~N} \mathrm{~m}^{-1}$; NanoWorld, Neuchatel, Switzerland) with a pre-attached glass microsphere (radius of curvature $R=5.6 \mu \mathrm{m}$ ) was used in all experiments; the use of a single probe was considered to reduce errors (ca. $15-20 \%$ ) introduced by cantilever spring constant $(k)$ measurement [52]. This property was determined prior to bio-indentation studies using the method of Hutter and Bechhoefer $\left(k=0.02876 \mathrm{~N} \mathrm{~m}^{-1}\right)$ [53] and obtaining a force vs. distance curve on the bottom of the glass Petri dish (in media) in a region devoid of cells. The AFM probe was lowered into the medium, positioned above the region of interest and an optical microscopy image was obtained for each cell. The probe was then brought into contact with the surface using the automated approach. Force vs. distance curves were obtained on nuclear and cytoplasmic (off-nuclei) regions (50 cells of each cell type, one force curve per region of the cell; $N=50 \times 2$ ). For the latter regions, the AFM probe did not 'reach over' the nuclei and therefore possible undesirable cantilever-nuclei interactions were avoided. An approach distance of $50 \mu \mathrm{m}$, a force set-point of $10 \mathrm{nN}$ (before releasing the applied load) and a tip velocity of $5 \mu \mathrm{m} \mathrm{s}^{-1}$ were used. Indentation speeds between 3 and 16 $\mu \mathrm{m} \mathrm{s}^{-1}$ have been shown to result in relatively constant $E$ values [54]. Force curve acquisition and data processing was performed using the AFM software (JPK Data Processing Software V5.1.13). Force curves (approach cycle) were off-set (baseline) and tilt corrected; the contact point (CP) in each curve was estimated by visual inspection (deviation from baseline) and heights corrected for cantilever bending (converted to tip-sample separation). $E$ values were then obtained by fitting the corrected approach force curves to a Hertzian model, which describes the variation of applied load $F$ with indentation $\delta$ as a sphere (radius $R$; at the end of a parabolic indenter) is pressed into an elastic half-space [55]:

$F=\frac{4 \sqrt{R}}{3\left(1-v^{2}\right)} E \delta^{3 / 2}$

where $v=$ Poisson ratio ( 0.5 , assuming cells to be flat, isotropic and incompressible, in keeping with most studies on soft biological systems [56]. 
Indentations initially were restricted to ca. $10 \%$ of the cell thickness (nuclear regions), since above this range, substrate effects make a large contribution to the apparent stiffness and the Hertz model may no longer be applied [54-57]. Thus, cell thickness measurements of wild type SNB-19 cells (same media and glass-bottomed petri dish) were obtained from AFM line-profiles (NanoWizard 3, JPK Instruments) using an 'arrow-shaped' Si cantilever (HQ:CSC37/No Al; probe 'C', nominal length $I_{\text {nom }}=300 \pm 5 \mu \mathrm{m}$, width $w_{\text {nom }}=35 \pm 3 \mu \mathrm{m}$, thickness $t_{\text {nom }}=2.0 \pm 0.5 \mu \mathrm{m}$, spring constant $k_{\text {nom }}=0.4 \mathrm{~N} \mathrm{~m}^{-1}$; Mikromasch Europe, Wetzlar, Germany) with integrated tip $(R=8 \mathrm{~nm}, h=12-18 \mu \mathrm{m} ; n=10)$. Cell thicknesses were $8.2 \pm 3.3$ and $1.1 \pm 0.4 \mu \mathrm{m}$ for nuclear and cytoplasm, respectively; $\delta$ values were restricted to $\mathrm{ca}$. $0.5 \mu \mathrm{m}$ from the $\mathrm{CP}$, which provided a good Hertzian model fit of the force-distance AFM data in the case of nuclear regions. The indentation of $\mathrm{ca} .50 \%$ of cell thickness for cytoplasmic areas yielded more stable Hertzian fit plots, although the resultant $E$ values agreed with those obtained at $10 \%$ indentation in most cases. Some force-curves ( $<5 \%)$, from all cell types, were rejected during acquisition due to nonfavourable tip-sample interactions (erroneously shaped curves). Differences in $E$ values associated with using the spherical indenter model rather than the chosen parabolic indenter model, which is sufficient for shallow contacts, were $<10 \%$, and within the error of accurately determining $k$.

Since CD44 siRNA knock-down was only partially complete, a proportion of the cells deemed to be knocked down were selected for further analysis. This was carried out by 3 of the investigators ( $Z M, Q A$ and $\mathrm{HF}$ ) independently examining the phase contrast images of each cell taken immediately before AFM data acquisition. This was done in a masked fashion with images not identifiable until after examination. Cells were only deemed knocked down when there was a $100 \%$ consensus of images where the morphology was similar to published reports [27].

\subsection{Statistics}

Migration and Flow analysis: Statistical analysis was performed using one-way ANOVA followed by Tukey's multiple comparison post-test with a probability $\alpha$ of less than 0.05 being regarded as significant. The software package GraphPad Prism 3.02 was used to calculate the statistical tests where all data are expressed as mean values.

AFM analysis: $E$ values (independent groups; nuclear and cytoplasm areas) were analysed using Q-Q plots and normality tests (Shapiro-Wilks; $\alpha=0.05$, two-tailed) were used to compare distributions [58]. Homogeneity was tested using a Levene's test ( $\alpha=0.05$, twotailed) and Student's t-tests ( $\alpha=0.05$, two-tailed) used to compare means [58]. All statistics were performed using SPSS Statistics 21 (IBM SPSS, NY, USA).

\section{Results}

Previously, we have shown that blocking CD44 expression in glioma cells resulted in a repression of cell invasion and this effect was mediated in part by RhoGTPase signalling [27]. In this report, we have extended these studies and examined the downstream effects of CD44 knock-down on the expression of cytoskeletal proteins involved in cellular migration and cellular stiffness $(E)$. SNB-19 glioma cells transfected with CD44 siRNA contained lower CD44 protein levels compared with those transfected with scrambled control siRNA (Fig. 1a). Functionally, SNB-19 cells transfected with CD44-targeted siRNA were less migratory than parental SNB-19 cells (Fig. $1 b, p<0.05$ ). 


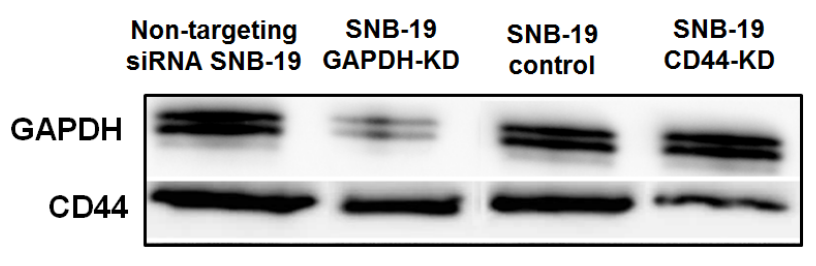

\section{a}

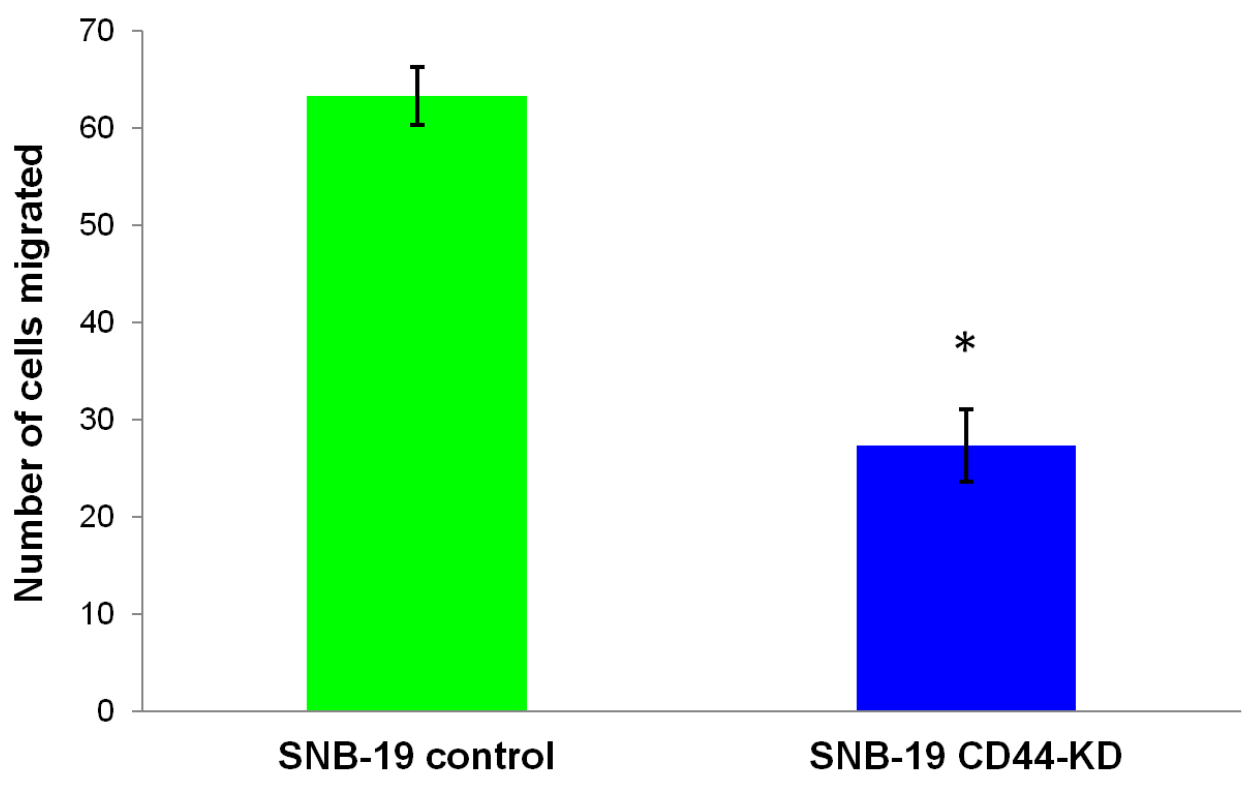

b

Fig. 1. CD44 knock-down (KD) reduces migratory potential of the SNB-19 cells. (a) Western blots of CD44 expression in non-targeting siRNA-, GAPDH-, parental controls and CD44-KD SNB-19 cells. Decreased expression level of CD44 in CD44-KD cells is demonstrated. Expression of GAPDH is also shown. (b) Significantly reduced migratory potential of the SNB-19 cells following CD44-KD was observed using the Transwell ${ }^{\mathrm{TM}}$ modified Boyden chamber technique, compared to the controls cells. * indicates statistical significance $(p<0.05)$.

In agreement with our previous study [27], knock-down of CD44 in SNB-19 cells resulted in a dramatic change in cellular morphology (Fig. 2a). In CD44 knock-down cells, the cells tended to appear rounder, lose the distinct CD44 positive staining and contain fewer processes. F-actin, vimentin, GFAP and microtubule positive staining was less pronounced in CD44 knock-down SNB-19 cells compared to control SNB-19 cells. While vimentin positive staining was associated with prominent filamentous staining in control cells, vimentin staining in CD44 knock-down cells appeared to be more granular and punctuated at focal adhesion points near edges of cells (Fig. 2, arrows). GFAP staining in CD44 knock-down cells seem to lose most of the central cytoplasmic positive staining. 
First online: 26 December 2015, pp. 1-11, doi: 10.1007/s12668-015-0189-2

www.link.springer.com/article/10.1007/s/12668-015-0189-2
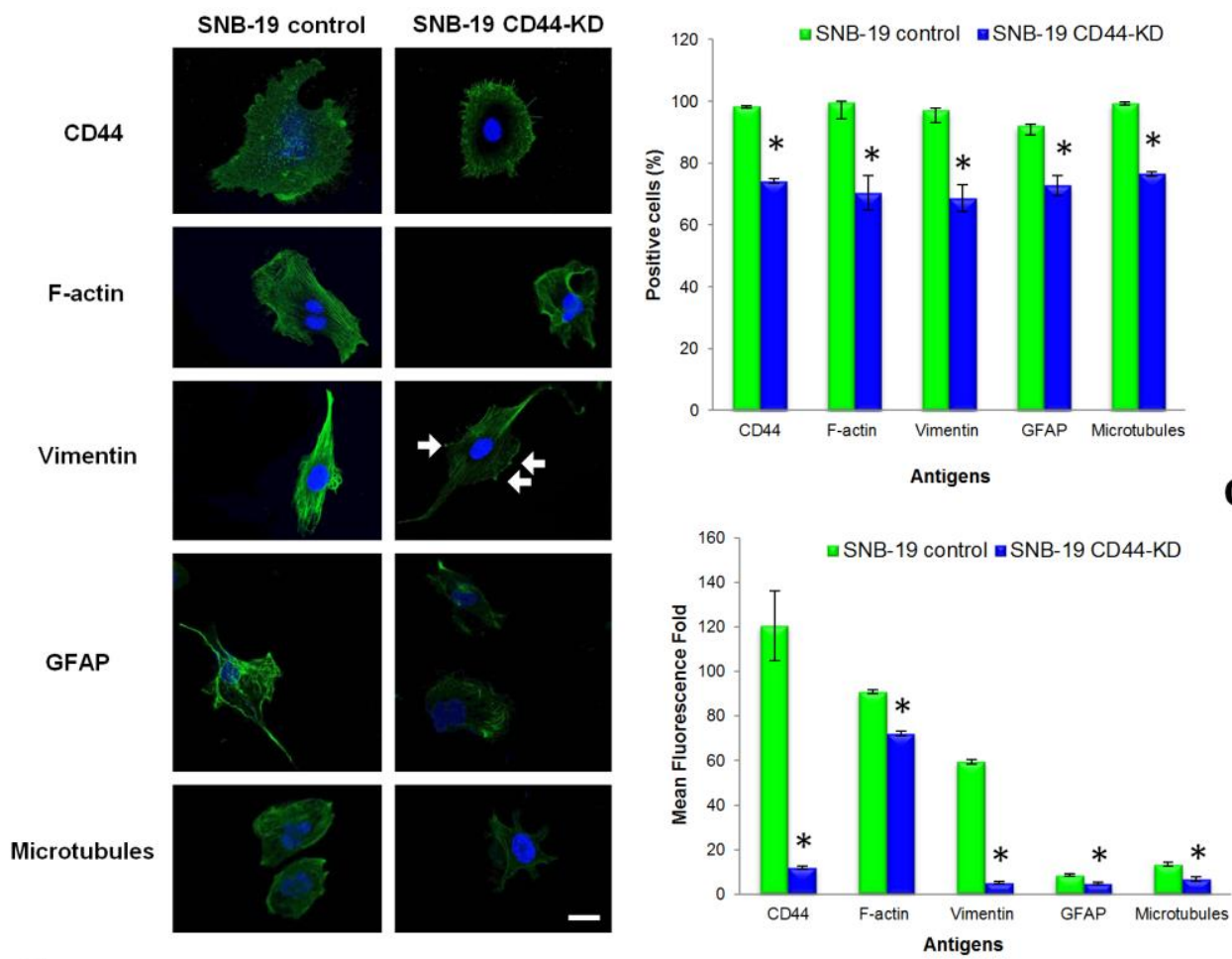

a
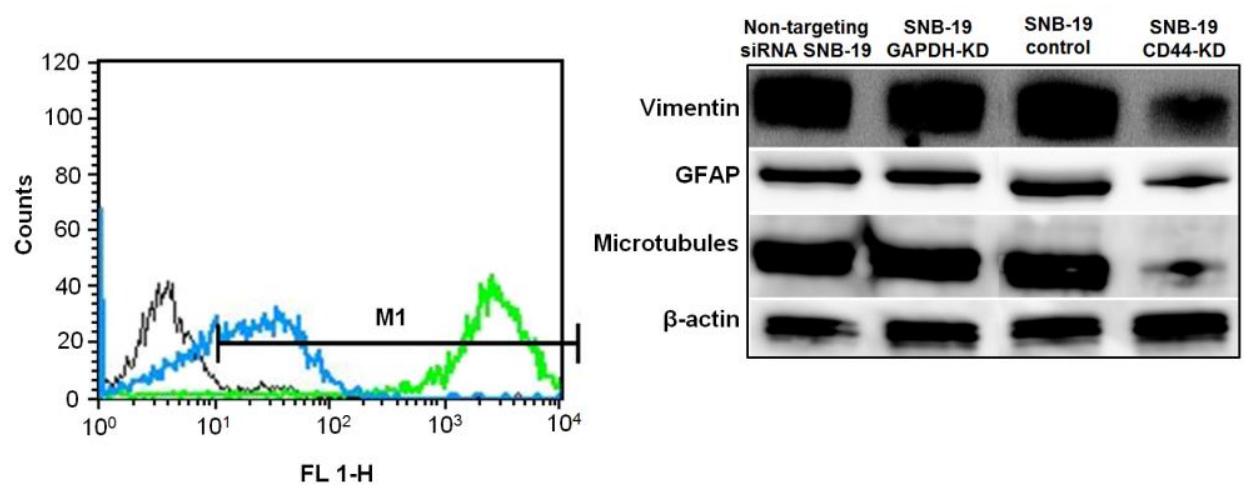

b

Fig. 2. Expression analyses of cytoskeleton-associated molecules in the SNB-19 cells. (a) Cellular morphology and distribution of CD44, F-actin, vimentin, GFAP and microtubules in SNB-19 cells and CD44-KD SNB-19 cells are illustrated by ICC staining. Antigenic proteins were stained by Alexa Fluor 488 (Green) with Hoechst Blue nuclei counterstain (Blue). Arrows mark focal adhesion points; 40x objective and scale bar $=10 \mu \mathrm{m}$ (all images). (b) Representative histogram of the flow cytometry analysis that shows a reduced percentage of CD44-expressing population in CD44-KD (Blue) SNB-19 cells compared to SNB-19 controls (Green) cells. Negative control (Black) is also shown in the graph where primary antibody was omitted. (c \& d) Significantly reduced expression levels of CD44, F-actin, vimentin, GFAP and microtubules in CD44-KD SNB-19 cells were confirmed by flow cytometry, as indicated by percentage of positive cells (c) and mean fluorescence fold (d). Results are representative of three independent experiments carried out in triplicate $(n=3)$. * indicates statistical significance $(p<0.05)$. (e) Western blots of vimentin, GFAP and microtubules in nontargeting siRNA-, GAPDH-, SNB-19, and CD44-KD SNB-19 cells. Decreased expression levels of vimentin, GFAP and microtubules in CD44-KD cells are demonstrated. Expression of $\beta$-actin (as loading control) is also shown. 


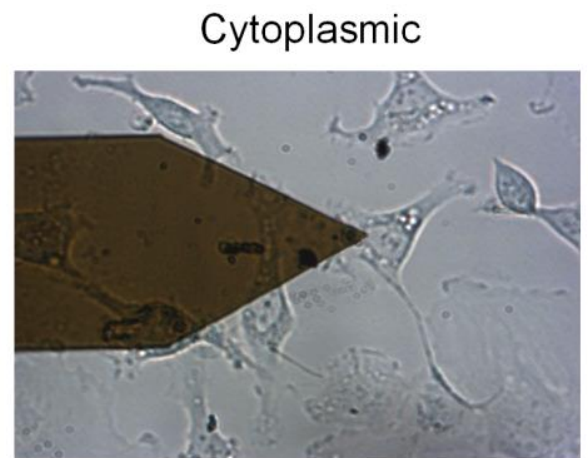

SNB-19 control

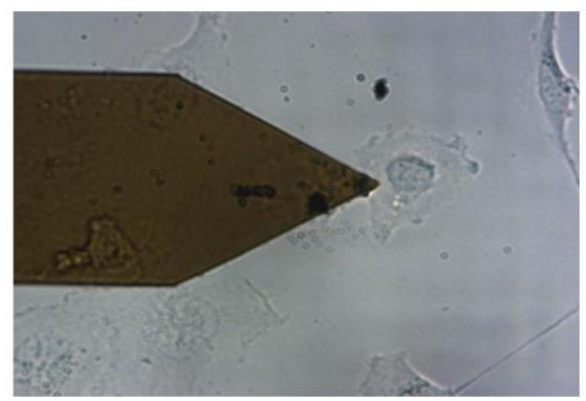

a

SNB-19 CD44-KD
Nuclear

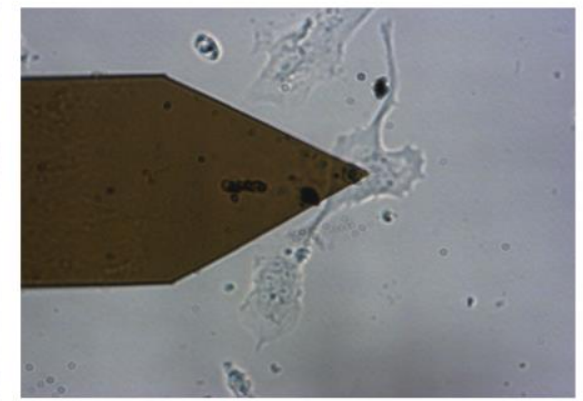

SNB-19 control

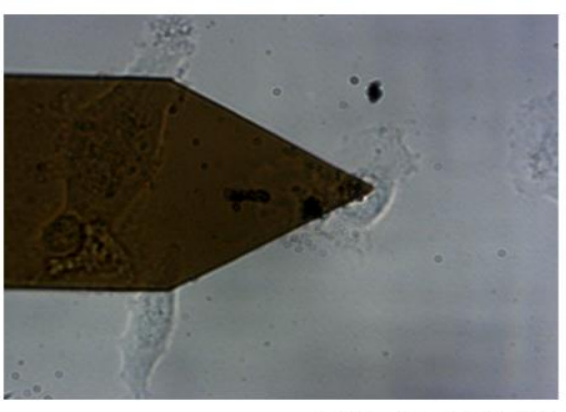

SNB-19 CD44-KD
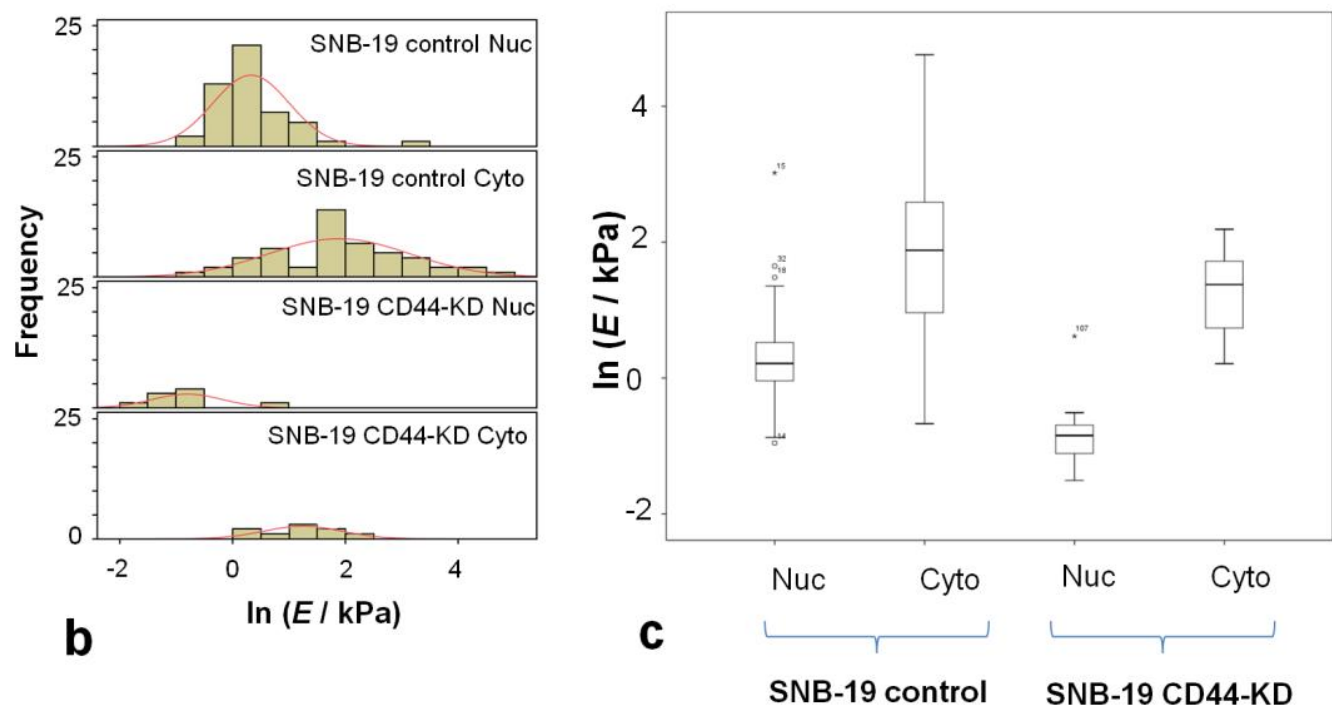

Fig. 3. AFM elasticity measurements of SNB-19 cells. (a) Phase images showing AFM nanoindentation analyses above cytoplasmic and nuclear areas of SNB-19 cells and CD44-KD SNB-19 cells. Cell types were differentiated by cellular morphology. (b \& c) Boxplots showing frequency (b) and mean (c) distributions of In $E$ obtained from SNB-19 cells and CD44-KD SNB-19 cells on nuclear (nuc) and cytoplasm (cyto) regions $(N=50 \times 4)$. For both cell types, $E$ values obtained from nuclear regions were significantly lower than those from cytoplasmic regions $(p<0.001)$. Focusing on nuclear regions, CD44-KD SNB-19 cells showed significantly lower $E$ values compared to SNB-19 cells ( $p<$ $0.001)$. 
Quantitative analysis using flow cytometric analysis demonstrated that the reduction in Factin, vimentin, GFAP and microtubule expression in SNB-19 CD44 knock-down cells was significant compared with wild type SNB-19 cells (Fig. 2c-d, $p<0.05$ ). Similar results demonstrating the reduction in vimentin, GFAP and microtubule expression in CD44 knockdown cells were seen in Western Blot analysis (Fig. 2e).

We next asked the question if the changes in morphology and reduced cytoskeletal protein expression and migration seen in CD44 knock-down SNB-19 cells was associated with a difference in mechanical properties, specifically, cell stiffness $(E)$. Prior to the analysis of cell stiffness, we needed to insure that the AFM measurements were conducted on cells with CD44 knocked down, as siRNA silencing of CD44 in SNB-19 cells did not result in a total reduction of CD44 protein (Fig. 1a). Images were taken of every cell in which $E$ values were determined (Fig. 3a). For identifying CD44 knock-down cells, three investigators independently scored all images in a blinded fashion and where there was $100 \%$ agreement on morphological changes associated with CD44 knock-down, these values were analysed. $E$ values of wild type SNB-19 and SNB-19/CD44 knock-down cells, from nuclear and cytoplasmic regions, were extracted from AFM force-distance curves (Fig. 3b). This property was found to be log-normally distributed, as observed elsewhere for other types of cancer cells [37]; AFM nanoindentation results show the area over the nucleus of cell lines examined were significantly more compliant (pliable) than their cytoplasmic regions (Fig. $3 c, p<0.001)$. The most striking difference was seen when comparing nuclear regions of parental cells to those of CD44 knock-down cells. CD44 knock-down SNB-19 cells $(E=0.56$ $\pm 0.50 \mathrm{kPa})$ were less stiff than wild type cells $(E=1.93 \pm 2.86 \mathrm{kPa} ; p<0.001)$.

\section{Discussion}

In this report, we have extended our studies exploring potential mechanisms involved in CD44-mediated glioma cell invasion by investigating changes in cytoskeletal proteins and biomechanical properties associated with inhibition of CD44 expression. Cell invasion involves the coordinated integration of many signals and while much is known concerning the role of specific genes and proteins and their associated signalling pathways, little is known on the influence of biophysical processes that may contribute to glioma invasion. These are important to consider as increased cell invasion is likely to include the ability of the cell to resist deformation and increase the rate of cytoskeletal dynamics $[4,5]$. This is not a novel concept as Herpers et al., in the mid-1980s, suggested that glioma cells are able to adapt to the microenvironment via changes in the cytoskeleton [59]. In this paper, we demonstrate that by knocking down CD44 expression in a glioma cell line (SNB-19) there is an associated decreased expression of the intermediate filament proteins, vimentin and GFAP and of microtubules. Cell migration was decreased in CD44 knock-down cells in agreement with published reports that show that inhibition of CD44 expression in glioma cells result in a decrease in glioma invasion [27]. We hypothesised that by knocking down CD44 and there would be an associated change in glioma cell deformity; specifically, that the CD44 knock-down cells would become less pliable (more stiff and increased $E$ values). Much of the published information concerning this area assumes that, in general, $E$ values of invasive cancer cells are lower (more pliable) than their normal cell counterparts [13]. In other words, the effect on reduced migration could be due, in part, to a change in mechanical stiffness and that by increasing cellular stiffness, invasion would be decreased. However, our results showed that CD44 knock-down glioma cells were less stiff than control cells. This difference was only seen in the area over the nucleus as there was no difference in cellular stiffness when AFM nanoindentation measurements were conducted away from the nucleus. Recently, Rathje et al. [60] reported that cellular stiffness was associated with 
the reorganisation of vimentin. Interestingly, vimentin is known to be perinuclear and its network extends throughout the cell during migration [61]. Rathje et al. showed that oncogenes can induce vimentin filament collapse and this is mediated by Histone deacetylase 6 (HDAC6), an enzyme responsible for deacetylation of histones resulting in condensed chromatin [60]. In our current work, one of the most apparent changes among the cytoskeletal proteins examined was seen in vimentin expression and its cellular distribution. We have previously shown that the functional effects of inhibiting CD44 are mediated, in part, by RhoGTPase signalling [27]. Transfection of U373 glioma cells with a constitutively active RhoGTPase resulted in an increase cellular stiffness, as measured by AFM nanoindentation [28]. We propose that in our experimental design, knockdown of CD44 leads to a decrease in vimentin and that the decrease in stiffness around the nucleus is in part due to the re-organisation of vimentin (Fig. 4). There is evidence that supports the idea that the nucleus can serve as a mechanosensor of external and internal forces $[62,63]$ and the tethering of chromatin to the nuclear envelope can affect nuclear stiffness [64]. Lamin proteins are key players that help connect chromatin and the cytoskeleton [65-67], and levels of lamin protein expression have been shown to influence cell migration and cell viability [67]. The role of the lamin proteins in mechanotransduction and nuclear function is a very exciting area of research and information from these investigations will provide much needed knowledge into the regulation of gene expression due to biomechanical and microenviornmental stresses in GBM.

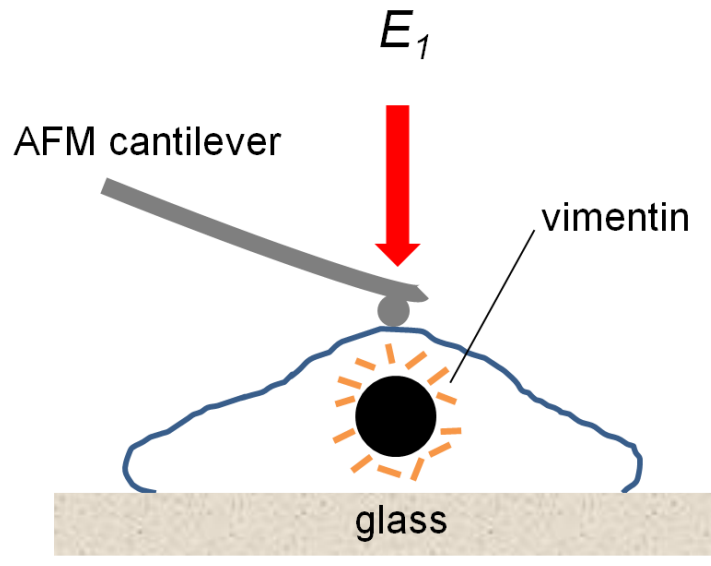

a

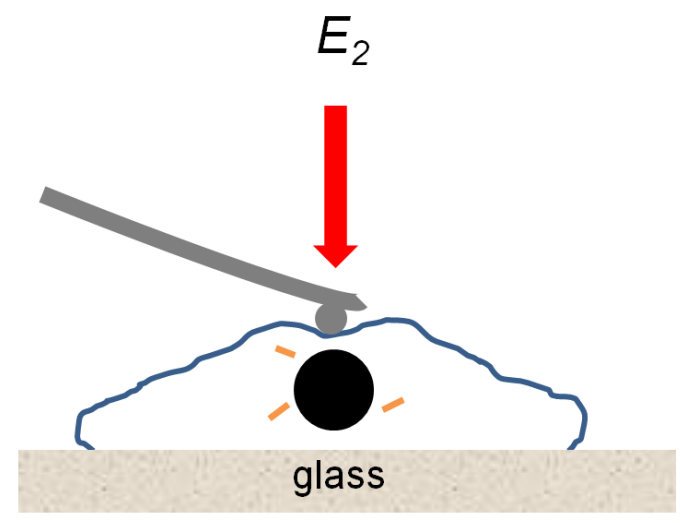

b

Fig. 4. Simplified model of the effect of CD44-KD on nuclear stiffness. Schematic diagram of our working model depicting a decrease in $E$ values caused by the reduced amounts and the reorganisation of perinuclear vimentin in CD44-KD SNB-19 cells (b) $\left(E_{2}\right.$, more compliant) compared with SNB-19 control cells $(\mathbf{a})\left(E_{1}\right)$.

As evidence increases as to the importance of a dynamic tumour microenvironment, the incorporation of mechanical properties and how tumour and host cells respond to external forces will be critical for a better understanding of host/tumour interactions. The cytoskeletal proteins allow the cell to adapt to microenvironmental changes. While cell deformation characteristics are determined by cytoskeletal dynamics [5], consideration of the factors that influence nanomechanical properties should be considered when making comparisons of observed $E$ values between studies. The cellular thickness, depth and 
duration of indentation, as well as the loading rate, can all affect $E$ values [6]. While there are many studies on different types of cancers that report that cancer cells are less stiff than less aggressive or non-neoplastic control cells, currently the strongest comparison that holds that cancer cells are less stiff than cells of same origin are in ovarian and bladder cancers [71]. Although our data demonstrate a decrease in glioma cell stiffness in less invasive cells, comparing the same isogenic cell line except for knocking down CD44, the general statement 'more aggressive, invasive cells are less stiff' probably does not hold true for all cancer cell types and is likely to be dependent on context and will vary depending on microenvironmental stresses. These issues should be considered for future studies on investigating the influence of biomechanical properties on glioma host/tumour interactions and glioma cell invasion.

Acknowledgements We thank Drs Robert Field and Alex Winkle from JPK Instruments, Cambridge, UK for loan of the CellHesion 200 and NanoWizard 3 AFM instruments and Dr Torsten Muller from JPK Instruments, Berlin, Germany, for initial AFM training. We also thank Brain Tumour Research and Institute for Biomedical and Biomolecular Sciences for support.

\section{References}

1. Rape A, Ananthanarayanan B, Kumar S (2014) Engineering strategies to mimic the glioblastoma microenvironment. Adv Drug Del Rev 79-80: 172-183.

2. Xie Q, Mittal S, Berens ME (2014) Targeting adaptive glioblastoma: an overview of proliferation and invasion. Neuro-Oncology 16: 1575-1584

3. Kim Y, Kumar S (2014) CD44-mediated adhesion to hyaluronic acid contributes to mechanosensing and invasive motility. Mol Cancer Res 12: 1416-1429.

4. Ketene AN, Schmelz EM, Roberts PC, Agah M (2012) The effects of cancer progression on the viscoelasticity of ovarian cell cytoskeleton structures. Nanomedicine 8: 93-102.

5. Babahosseini H, Ketene AN, Schmelz EM, Roberts PC, Agah M (2014) Biomechanical profile of cancer stem-like/tumor-initiating cells derived from a progressive ovarian cancer model. Nanomedicine 10: 1013-1019.

6. Lekka M, Gil D, Pogoda K, Dulińska-Litewka J, Jach R, Gostek J, Klymenko O, PrauznerBechcicki S, Stachura Z, Wiltowska-Zuber J, Okoń K, Laidler P (2012) Cancer cell detection in tissue sections using AFM. Arch Biochem Biophys 518: 151-156.

7. Swaminathan V, Mythreye K, O'Brien ET, Berchuck A, Blobe GC, Superfine R (2011) Mechanical stiffness grades metastatic potential in patient tumor cells and in cancer cell lines. Cancer Res 71: 5075-5080.

8. Yamazaki D, Kurisu S, Takenawa T (2005) Regulation of cancer cell motility through actin reorganization. Cancer Sci 96: 379-386.

9. Rao J, Li N (2004) Microfilament actin remodeling as a potential target for cancer drug development. Curr Cancer Drug Targets 4: 345-354.

10. Weder G, Hendriks-Balk MC, Smajda R, Rimoldi D, Liley M, Heinzelmann H, Meister A, Mariotti A (2014) Increased plasticity of the stiffness of melanoma cells correlates with their acquisition of metastatic properties. Nanomedicine 10: 141-148.

11. McKnight AL, Kugel JL, Rossman PJ, Manduca A, Hartmann LC, Ehman RL (2002) MR elastography of breast cancer: preliminary results. AJR Am J Roentgenol 178: 1411-1417.

12. Bercoff J, Chaffai S, Tanter M, Sandrin L, Catheline S, Fink M, Gennisson JL, Meunier M (2003) In vivo breast tumor detection using transient elastography. Ultrasound Med Biol 29: 13871396.

13. Suresh S (2007) Biomechanics and biophysics of cancer cells. Acta Biomater 3: 413-438.

14. Birch M, Mitchell S, Hart IR (1991) Isolation and characterization of human melanoma call variants expressing high and low levels of CD44. Cancer Res 51: 6660-6667.

15. Sy MS, Guo YJ, Stamenkovic I (1991) Distinct effects of 2 CD44 isoforms on tumour-growth in vivo. J Exp Med 174: 859-866. 
16. Okada H, Yoshida J, Sokabe M, Wakabayashi T, Hagiwara M (1991) Supression of CD44 expression decreases migration and invasion of human glioma cells. Int J Cancer 66: 255-260.

17. Gunia S, Hussein S, Radu DL, Putz KM, Breyer R, Hecker H (1999) CD44s-targeted treatment with monoclonal antibody blocks intercerebral invasion and growth of $9 \mathrm{~L}$ gliosarcoma. Clin Exp Metastasis 17: 221-230.

18. Stern R, Shuster S, Wiley TS, Formby B (2001) Hyaluronidase can modulate expression of CD44. Exp Cell Res 266: 167-319.

19. Wiranowska M, Ladd S, Moscinski LC, Hill B, Haller E, Mikecz K, Plaas A (2010) Modulation of hyaluronan production by CD44 positive glioma cells. International J Cancer 127: 532-542.

20. Pietras A, Katz AM, Ekström EJ, Wee B, Halliday JJ, Pitter KL, Werbeck JL, Amankulor NM, Huse JT, Holland EC (2014) Osteopontin-CD44 signaling in the glioma perivascular niche enhances cancer stem cell phenotypes and promotes aggressive tumor growth. Cell Stem Cell 14: 357-369.

21. Misra S, Hascall VC, De Giovanni C, Markwald RR, Ghatak S (2009) Delivery of CD44 shRNA/nanoparticles within cancer cells. J Biol Chem 284: 12432-12446.

22. Stamenkovic I, Aruffo A, Amiot M (1991) The hematopoietic and epithelial forms of CD44 are distinct polypeptides with different adhesion potentials for hyaluronate-bearing cells. EMBO J 10: 343-348.

23. Delpech B, Maingonnat C, Girard N, Chauzy C, Maunoury R, Olivier A (1993) Hyaluronan and hyaluronectic in the extracellular matrix of human brain tumour stroma. Eur J Cancer 29A: 1012-1017.

24. Merzak A, Pilkington GJ (1997) Molecular and cellular pathology of intrinsic brain tumours. Cancer Metastasis Rev 16: 155-177.

25. Günthert U, Hofmann M, Rudy W, Reber S, Zöller M, Haussmann I, Matzku S, Wenzel A, Ponta $H$, Herrlich P (1991) A new variant of glycoprotein CD44 confers metastatic potential to rat carcinoma cells. Cell 65: 13-24.

26. Okamoto I, Tsuiki H, Kenyon LC, Godwin AK, Emlet DR (2002) Proteolytic Cleavage of the CD44 adhesion molecule in multiple human tumours. Am J Pathol 160: 441-447.

27. Maherally Z, Smith JR, An Q, Pilkington GJ (2012) Receptors for hyaluronic acid and poliovirus: A combinatorial role in glioma invasion? PLoS One 7: e30691.

28. Mackay JL, Keung AJ, Kumar S (2012) A genetic strategy for the dynamic and graded control of cell mechanics, motility, and matrix remodelling. Biophys J 102: 434-442.

29. Wang B, Lancon P, Bienvenu C, Vierling P, Di Giorgio C, Bossis G (2013) A general approach for the microrheology of cancer cells by atomic force microscopy. Micron 44: 287-297.

30. Vadillo-Rodriguez V, Dutcher JR (2009) Dynamic viscoelastic behaviour of individual Gramnegative bacterial cells. Soft Matter 5: 5012-5019.

31. Eaton P, West P (2010) Atomic Force Microscopy. Oxford Univ. Press, Oxford.

32. Binnig G, Quate CF, Gerber C (1986) Atomic force microscope. Phy Rev Lett 56: 930-903.

33. Sokolov I, Iyer S, Woodworth CD (2006) Recovery of elasticity of aged human epithelial cells in vitro. Nanomed Nanotechnol Biol Med 2: 31-36.

34. Finke M, Hughes JA, Parker DM, Jandt KD (2001) Mechanical properties of in situ demineralised human enamel measured by AFM nanoindentation. Surface Sci 491: 456-467.

35. Lekka M, Laidler P, Gil D, Lekki J, Stachura Z, Hrynkiewicz AZ (1999) Elasticity of normal and cancerous human bladder cells studied by scanning force microscopy. Eur Biophys J 28: 312316.

36. Chen B, Wang Q, Han L (2004) Using the atomic force microscope to observe and study the ultrastructure of the living BIU-87 cells of the human bladder cancer. Scanning 26: 162-166.

37. Cross SE, Jin YS, Rao J, Gimzewski JK (2007) Nanomechanical analysis of cells from cancer patients. Nat Nanotechnol 2: 780-783.

38. Cross SE, Jin YS, Tondre J, Wong R, Rao J, Gimzewski JK (2008) AFM-based analysis of human metastatic cancer cells. Nanotechnology 19: 384003.

39. Li QS, Lee GY, Ong CN, Lim CT (2008) AFM indentation study of breast cancer cells. Biochem Biophys Res Commun 374: 609-613.

40. Plodinec M, Loparic M, Monnier CA, Obermann EC, Zanetti-Dallenbach R, Oertle P, Hyotyla JT, Aebi U, Bentires-Alj M, Lim RY, Schoenenberger CA (2012) The nanomechanical signature of breast cancer. Nat Nanotechnol 7: 757-765.

41. Yokokawa MK, Takeyasu, Yoshimura SH (2008) Mechanical properties of plasma membrane and nuclear envelope measured by scanning probe microscope. J Microsc 232: 82-90. 
42. Fuhrmann A, Staunton JR, Nandakumar V, Banyai N, Davies PCW, Ros R (2011) AFM stiffness nanotomography of normal, metaplastic and dysplastic human esophageal cells. Phys Biol 8: 015007.

43. Zhang G, Long M, Wu ZZ and Yu WQ (2002) Mechanical properties of hepatocellular carcinoma cells. World J Gastroenterol 8: 243-246.

44. Gang Z, Qi Q, Jing C, Wang CY (2009) Measuring microenvironment mechanical stress of rat liver during diethylnitrosamine induced hepatocarcinogenesis by atomic force microscope. Microsc Res Techn 72: 672-678.

45. Xu W, Mezencev R, Kim B, Wang L, McDonald J, Sulchek T (2012) Cell stiffness is a biomarker of the metastatic potential of ovarian cancer cells. PLoS One 7(10): e46609.

46. Li YX, Schnekenburger J, Duits MHG (2009) Intracellular particle tracking as a tool for tumor cell characterization. J Biomed Optics 14: 064005.

47. Moreno-Flores S, Benitez R, Vivanco MD, Toca-Herrera JL (2010) Stress relaxation and creep on living cells with the atomic force microscope: a means to calculate elastic moduli and viscosities of cell components. Nanotechnology 21: 445101.

48. Faria EC, Ma N, Gazi E, Gardner P, Brown M, Noel NW, Snook RD (2008) Measurement of elastic properties of prostate cancer cells using AFM. Analyst 133: 1498-1500.

49. Docheva D, Padula D, Schieker M, Clausen-Schaumann H (2010) Effect of collagen I and fibronectin on the adhesion, elasticity and cytoskeletal organization of prostate cancer cells. Biochem Biophys Res Commun 402: 361-366.

50. Prabhune M, Belge G, Dotzauer A, Bullerdiek J, Radmacher M (2012) Comparison of mechanical properties of normal and malignant thyroid cells. Micron 43: 1267-1272.

51. An Q, Fillmore HL, Vouri M, Pilkington GJ (2014) Brain tumour cell line authentication, an efficient alternative to capillary electrophoresis by using a microfluidics-based system. NeuroOncology 16: 265-273.

52. Clifford CA, Seah MP (2005) Quantification issues in the identification of nanoscale regions of homopolymers using modulus measurement via AFM nanoindentation. Appl Surface Sci 252: 1915-1933.

53. Hutter JL, Bechhoefer J (1993) Calibration of atomic-force microscope tips. Rev Sci Instrum 64: 1868-1873.

54. McPhee G, Dalby MJ, Riehle M, Yin HB (2010) Can common adhesion molecules and microtopography affect cellular elasticity? A combined atomic force microscopy and optical study. Med Biol Eng Comp 48: 1043-1053.

55. Hertz H (1882) Ueber die Berührung fester elastischer Körper. J. fuer die Reine Angew. Math. 1882: 156-171.

56. Touham A, Nysten B, Dufrene YF (2003) Nanoscale mapping of the elasticity of microbial cells by atomic force microscopy. Langmuir 19: 4539-4543.

57. Dimitriadis EK, Horkay F, Maresca J, Kachar B, Chadwick RS (2002) Determination of elastic moduli of thin layers of soft material using the atomic force microscope. Biophys J 82: 27982810.

58. Pett MA (1997) Nonparametric Statistics for Health Care Research. SAGE Publications Inc., Thousand Oaks, California.

59. Herpers MJ, Budka H, McCormick D (1984) Production of glial fibrillary acidic protein (GFAP) by neoplastic cells: adaptation to the microenvironment. Acta Neuropathol 64: 333-338.

60. Rathje LZ, Nordgren N, Pettersson T, Rönnlund D, Widengren J, Aspenström P, Gad AKB (2014) Oncogenes induce a vimentin filament collapse mediated by HDAC6 that is linked to cell stiffness. Proc Natl Acad Sci USA 11: 1515-1520.

61. Chang L, Goldman RD (2004) Intermediate filaments mediate cytoskeletal crosstalk. Mol Cell Biol 5: 601-613.

62. Tai PW, Zaidi SK, Wu H, Grandy RA, Montecino M, van Wijnen AJ, Lian JB, Stein GS, Stein JL (2014) The dynamic architectural and epigenetic nuclear landscape: Developing the genomic almanac of biology and disease. J Cell Physiol 229: 711-727.

63. Gorjanacz M (2014) Nuclear assembly as a target for anti-cancer therapies. Nucleus 5: 47-55.

64. Schreiner, SM, Koo PK, Zhao Y, Mochrie SGJ, King MC (2015) The tethering of chromatin to the nuclear envelope supports nuclear mechanics. Nat Commun 6: 7159-7171.

65. Osmanagic-Myers S, Dechat $T$, Foisner R (2015) Lamins at the crossroads of mechanosignaling. Genes Develop 29:225-237. 
66. Fedorchak GR, Kaminski A, Lammerding J (2014) Cellular mechanosensing: Getting to the nucleus of it all. Prog Biophys Mol Biol 115: 76-92.

67. Swift J, Discher DE (2014) The nuclear lamina is mechano-responsive to ECM elasticity in mature tissue. J Cell Sci 127: 3005-3015.

68. Harada T, Swift J, Irianto J, Shin J-W, Spinler KR, Athirasala A, Diegmiller R, Dingal PCDP, Ivanovska IL, Discher DE (2014) Nuclear lamin stiffness is a barrier to 3D migration, but softness can limit survival. J Cell Biol 204: 669-682.

69. Ramos JR, Rabijan J, Garcia R, Lekka M (2014) The softening of human bladder cancer cells happens at an early stage of the malignancy process. Beilstein J Nanotechnol 5: 447-457.

70. Ferrera D, Canale C, Marotta R, Mazzaro N, Gritti M, Mazzanti M et al. (2014) Lamin B1 overexpression increases nuclear rigidity in autosomal dominant leukodystrophy fibroblasts. FASEB J 28: 3906-3918.

71. Ramos JR, Rabijan J, Garcia R, Lekka M (2014) The softening of human bladder cancer cells happens at an early stage of the malignancy process. Beilstein J Nanotechnol 5: 447-457. 